\title{
Effect of Polydextrose Intake on Constipation in Japanese Dialysis Patients: A Triple-Blind, Randomized, Controlled Trial
}

\author{
Mikiko SHIMADA $^{1,2}$, Nobuo NAGANO ${ }^{3}$, Saori GOTO ${ }^{4}$, Kyoko ITO ${ }^{4}$, Takaaki TsuTSUI ${ }^{3}$, \\ Tetsuo ANDO ${ }^{3}$, Hiroharu KAMIOKA ${ }^{2}$ and Tetsuya OGAWA ${ }^{5}$ \\ ${ }^{1}$ Medical Corporations Hidaka Rehabilitation Hospital, 2204 Yoshii, Takasaki, Gunma 370-2104, Japan \\ ${ }^{2}$ Graduate School of Agriculture, Tokyo University of Agriculture, 1-1-1 Sakuragaoka, \\ Setagaya-ku, Tokyo 156-8502, Japan \\ ${ }^{3}$ Medical Corporations Hidaka Hospital, Takasaki, 886 Nakao, Takasaki, Gunma 370-0001, Japan \\ ${ }^{4}$ Medical Corporations Heisei Hidaka Clinic, 807-1 Nakao, Takasaki, Gunma 370-0001, Japan \\ ${ }^{5}$ Department of Medicine, Medical Center East, Tokyo Women's Medical University, \\ 2-1-10 Nishiogu, Arakawa, Tokyo 116-8567, Japan
}

(Received February 9, 2015)

\begin{abstract}
Summary The objective of the present study was to evaluate bowel habits induced by ingestion of $10 \mathrm{~g}$ polydextrose (PDX) fed to Japanese hemodialysis (HD) patients. This was a randomized, placebo-controlled, triple-blind, parallel-group controlled, 8-wk study. A total of $50 \mathrm{HD}$ outpatients capable of self-management (51-79 y of age) were recruited at $\mathrm{H}$ Clinic, Japan. Inclusion criteria for participation in the study were ingestion of one or more laxative tablets for more than 3 mo and having received HD for more than 6 mo. The participants were randomly assigned to 2 groups: A (0 g polydextrose/d; control), B ( $10 \mathrm{~g}$ polydextrose/d; PDX). The primary outcome measure was stool frequency. Secondary outcomes were stool consistency, abdominal pain, intestinal bloating and clinical biochemistry indexes. PDX had no significant effect on blood biochemistry indexes. The PDX group showed significant improvements in bowel function (stool frequency increased from 3.0 times per week to 7.5 times per week) and reported no laxation problems (abdominal distention, cramps, and diarrhea) $(p<0.01)$. Regular consumption of the PDX products increased dietary fiber intake to recommended levels and improved bowel habits.
\end{abstract}

Key Words polydextrose, dietary fiber, stool frequency, hemodialysis, constipation

Dietary fiber was initially defined by Trowell and Burkitt as "remnants of plant cell walls which were not hydrolyzed and digested by human enzymes" (1). Dietary fiber has a broad range of beneficial health applications such as the treatment of colonic disorders, lowering the risk of heart disease, diabetes and colon cancer, improving measures of glycemic control and gastrointestinal function, and other effects (2-6). Polydextrose (PDX) is a water-soluble polymer of glucose that provides to foods the bulk and texture of sucrose, and thus gives it the properties of dietary fiber. It was developed as a sugar replacement to lower the energy value of food, and at only one-quarter the caloric value (7). PDX was approved as a direct food additive in 1987 by the US Food and Drug Administration for use as a nutrient supplement, texturizer, stabilizer or thickener, formulation acid and humectant. PDX has been approved as a dietary fiber in a growing number of countries including Japan, Argentina, Belgium, Finland, Norway, France, Australia, and New Zealand. PDX has been reported to increase fecal bulk, soften stools and lower fecal $\mathrm{pH}$ due to its partial fermentability in the large bowel $(8-11)$. Constipation is defined by the new Roma III criteria (12,

E-mail:m2r2.smd@flower.email.ne.jp
13). It is estimated that $12 \%$ of people worldwide are suffering from self-defined constipation, and a third of the population in western industrial countries suffers from constipation at least from time to time (14). Constipation is a more common problem for patients receiving hemodialysis (HD), although the increased incidence of constipation in long-term dialysis patients is based on self-reported data (15). Some studies have shown that $53 \%$ ( $8 \%$ to $57 \%$ ) of patients with end-stage renal disease who are receiving dialysis suffer from constipation $(16,17)$. Many high-fiber foods are also high in potassium and phosphorus and therefore must be restricted in the diet. In addition, many medications that HD patients take can cause constipation. Intervention studies utilizing PDX have seldom been conducted in Japan, which prompted us to perform the current study to examine if our newly devised dietary fiber jellies containing $5 \mathrm{~g}$ PDX per piece would improve constipation in our HD patients.

\section{Materials and Methods}

Participants and polydextrose intake. A total of $50 \mathrm{HD}$ outpatients capable of self-management (51-79 y of age) were recruited at $\mathrm{H}$ Clinic, Japan. There were 34 males and 16 females with average ages of 67.8 and 
Table 1. Clinical characteristics of participants in before ingestion.

\begin{tabular}{|c|c|c|c|c|}
\hline & \multicolumn{4}{|c|}{ Before ingestion } \\
\hline & \multicolumn{2}{|c|}{ Enrolled } & \multicolumn{2}{|c|}{ Analyzed } \\
\hline & Placebo & PDX & Placebo & PDX \\
\hline$n$ & 25 & 25 & 13 & 16 \\
\hline Age & $67.4 \pm 8.2$ & $62.3 \pm 8.0$ & $66.1 \pm 9.4$ & $67.5 \pm 8.5$ \\
\hline BMI $\left(\mathrm{kg} / \mathrm{m}^{2}\right)$ & $21.8 \pm 3.2$ & $22.5 \pm 4.7$ & $22.9 \pm 3.8$ & $21.3 \pm 4.2$ \\
\hline Diabetes mellitus & $15(60 \%)$ & $14(56 \%)$ & $9(69 \%)$ & $9(56 \%)$ \\
\hline Gender (male/female) & $(17 / 8)$ & $(17 / 8)$ & $(9 / 4)$ & $(10 / 6)$ \\
\hline Dialysis duration (y) & $9.4 \pm 8.0$ & $9.3 \pm 6.8$ & $9.3 \pm 10.0$ & $9.0 \pm 6.9$ \\
\hline Defecation frequency (/wk) & $3.3[2.5-7.0]$ & $4.0[3.0-7.0]$ & $3.5[2.5-7.0]$ & $3.0[2.5-7.0]$ \\
\hline Stool score & $2.9 \pm 1.3$ & $3.2 \pm 1.1$ & $3.1 \pm 0.3$ & $3.2 \pm 0.3$ \\
\hline
\end{tabular}

Mean \pm SD or median [25th percentile- 75 th percentile].

Two-sample $t$-test for continuous variables and Fisher's exact test for categorical variables.

No significant changes were observed between the PDX group or the placebo group.

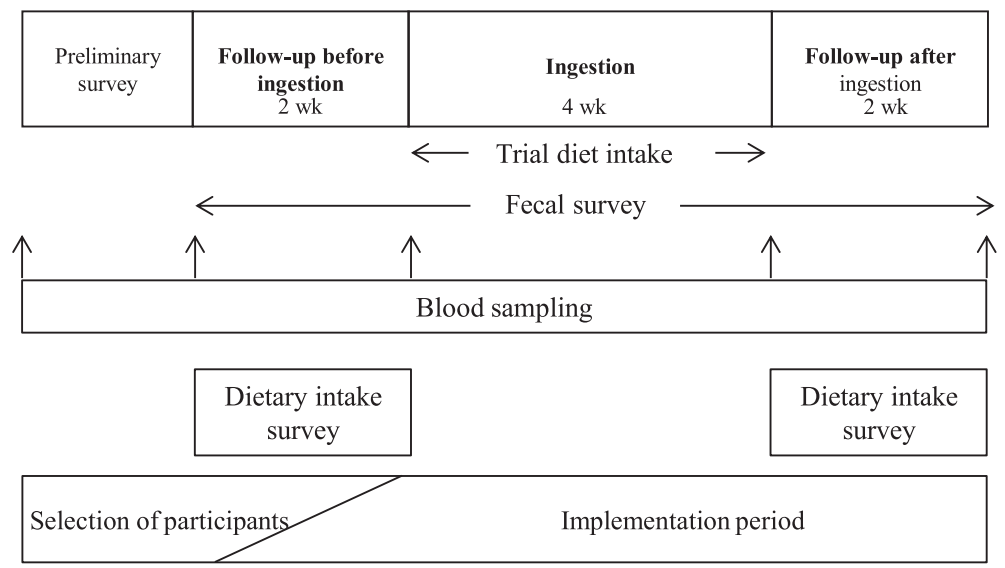

Fig. 1. Study design. Candidates were selected by a preliminary survey. Assessment test periods were as follows: follow-up before ingestion ( $2 \mathrm{wk})$, ingestion ( $4 \mathrm{wk})$, follow-up after ingestion ( $2 \mathrm{wk})$.

66.3 y, respectively (Table 1). After it was approved by the Ethical Review Board of the medical corporation $\mathrm{H}$ society, the study was conducted with appropriate safety considerations. In addition, in conformity with the Declaration of Helsinki, study participants received an explanation of the study purpose and methods, which was presented from June, 2013 to August, 2013. Written informed consent was obtained from all participants.

Inclusion criteria. Inclusion criteria for participation in the study were: (1) a signed consent form; (2) age 50-80 y inclusive; (3) ingestion of one or more laxative tablets for more than 3 mo; and (4) having received HD for more than 6 mo.

Exclusion criteria. Subjects with a history of severe gastrointestinal disorders, a history of adverse reactions to dietary fiber products, significantly poor patient weight (body weight less than 3 mo ago was $-3 \%$ ), interdialytic weight gain/dry weight (IDWG $>5 \%$ ), a random blood glucose level of $200 \mathrm{mg} / \mathrm{dL}$ (11.1 mmol/L) or higher, diet management, dementia disorder, taking supplements or food containing a large amount of lactic acid bacteria and bifidobacteria, participation in other clinical studies and clinical research, or inappropriate physician diagnosis were excluded. Intake of an experimental drug within 4 wk before study entry, participation in prebiotic, probiotic or laxative studies within 3 mo before study entry, use of antibiotics within 1 mo before study entry, and diarrhea or other chronic gastrointestinal complaints (e.g., irritable bowel syndrome) were all exclusion criteria. After the start of the study, after any intake of prebiotics, probiotics (live yoghurts, fermented milk drinks) and drugs active on gastrointestinal motility, phosphate binders, antibiotic treatment, or any class of laxative was not permitted. All medications taken throughout the duration of the study were recorded in diaries.

Study design. This was a randomized, placebo-controlled, triple-blind, parallel-group controlled, 8-wk study. Participant recruiting took place at the $\mathrm{H}$ Clinic in May 2013. The registration number of the study was TRC-13. The study was also registered as a University Hospital Medical Information Network Clinical Trials 
Table 2. Profile of test sample.

Placebo

erythritol, apple juice, brown rice black vinegar, agar, reduced sugar syrup, acidifier, gelatinizer, flavor, glycine, sweetening (acesulfame K, sucralose)
PDX

polydextrose, erythritol, apple juice, brown rice black vinegar, agar, reduced sugar syrup, acidifier, gelatinizer, flavor, glycine, sweetening (acesulfame K, sucralose)

$\begin{array}{llc}\text { Nutrition information } & & 11.7 \\ \text { Energy (kcal) } & 6.9 & 0 \\ \text { Protein (g) } & 0 & 0 \\ \text { Lipid (g) } & 0 & 2.8 \\ \text { Carbohydrate (g) } & 1.6 & 5 \\ \text { Dietary fibers (g) } & 0.1 & 7.6 \\ \text { Sodium (mg) } & 7.6 & 3.2 \\ \text { Potassium (mg) } & 3.2 & 0.4 \\ \text { Calcium (mg) } & 0.4 & 0.3 \\ \text { Phosphorus (mg) } & 0.3 & \end{array}$

The placebo included the same ingredients as the PDX jelly but was free of polydextrose (PDX).

Registry (UMIN-CTR) Clinical Study (Unique trial Number: 000011380) on 5 August 2013 (UMIN-CTRURL: http://www.umin.ac.jp/ctr/index.htm). The results are reported according to a CONSORT flow chart (Fig. 1).

For a period of $14 \mathrm{~d}$ before the dietary intervention study, participants were encouraged to follow a healthy lifestyle regimen and were advised not to make any significant changes to their diet, fluid intake, or routine physical activity during the 8-wk period of the study protocol. In that period, every day the subject was required to record information such as defecation frequency and stool score, and the subject was also interviewed about the amount of their activity during an average of $1 \mathrm{wk}$ prior to dialysis treatment day. After a 2-wk lead-in period, the eligible participants were assigned to either a PDX group (10 g polydextrose/d) or a placebo group $(0 \mathrm{~g}$ polydextrose/d) using a stratified randomization to guarantee comparability of the distribution of age, sex, HD history, diabetes, and quantity of laxative levels between the 2 groups. The randomization code was prepared by a staff member who was not involved in running the study, by using computer-generated random numbers.

Blinding. A statistician, who was not directly involved in the establishment of the groupings or the design of the study, was provided with the codes and the data for analysis. The group allocation was blinded for investigators (health care providers), outcome assessors and participants (triple-blind). The principal investigator was provided with a sealed envelope for each participant, but asked to open it only after entering all data into the computer or in a medical emergency. Allocation concealment was maintained successfully as no sealed envelope was opened voluntarily or accidentally during the study.

All test products were packaged, labelled, coded and randomized by a registered dietician at another facility before the study. The sample of PDX jelly or placebo (Table 2) was provided for the participants in sealed packages every $2 \mathrm{wk}$, and the PDX jelly and placebo samples were distributed by a registered dietician and nurse. The participants were asked to take one pack, twice a day, consisting of a total of 2 packs ( $40 \mathrm{~g}$ ) a day for $4 \mathrm{wk}$, preferably after breakfast and dinner. The dose for the PDX was chosen based on the commercially recommended level.

The participants in the PDX group took 2 packs of PDX jelly, which contained $5 \mathrm{~g}$ of PDX per pack, daily for $4 \mathrm{wk}$ and then underwent a washout period of $2 \mathrm{wk}$. Likewise, the participants in the placebo group took 2 packs of placebo jelly daily for 4 wk followed by a washout period of 2 wk. During the 2 -wk washout period, no study product was consumed. The feeding phase, including PDX intake, began on day 15 and continued for $28 \mathrm{~d}$. Body weight was measured on HD day. Clinical chemistry indexes were completed on days 0,14 and 42. Participants were asked to keep diaries every day throughout the study to record stool frequency, stool consistency according to the Bristol stool scale form (18, 19) (the modified score 1-6), abdominal pain (yes/no), unusual intestinal bloating (yes/no), and flatulence (yes/ no). Any concomitant medications, laxative use, intake of jelly, or adverse events were also recorded. To assess nutrient intake and monitor dietary compliance, food quality scores and 7-d food investigations were collected both at the beginning and end of the study. Dietary intake was assessed by using FFQg Ver. 4.5, developed by Yoshimura (Kenpaku-sha, Tokyo, Japan) (20).

Outcome measures. The primary outcome measure was stool frequency. Secondary outcomes were stool consistency, abdominal pain, intestinal bloating and clinical biochemistry indexes. Clinical biochemistry indexes were measures of liver and renal function, blood electrolytes, fasting blood glucose, triglyceride, cholesterol, hemoglobin, hematocrit and serum albumin. All comparisons were between treatment with PDX and the placebo.

Sample size estimation. Sample size estimation de- 


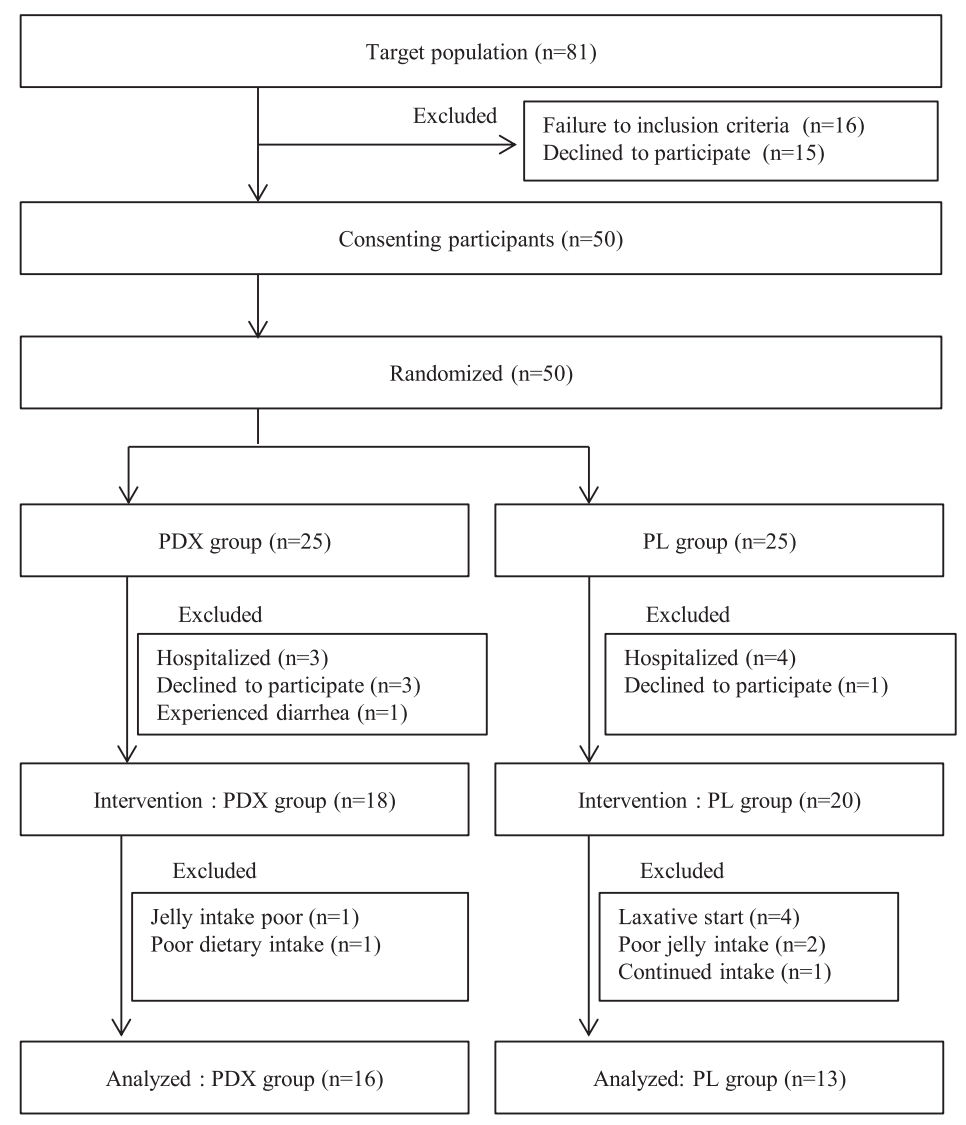

Fig. 2. Flowchart. A total of 50 participants were enrolled and randomized either to the PDX group $(n=25)$ or the placebo group $(n=25)$. Of the participants enrolled, 21 were not included for the efficacy analysis, primarily owing to protocol inclusion/exclusion violations in the placebo group $(n=12)$ and the use of treatment regimens such as laxatives or phosphate binders that were violations of the protocol in the PDX group $(n=9)$. The remaining 29 participants constituted the per protocol population, which was the primary efficacy population for the analysis.

Table 3. Defecation frequency.

\begin{tabular}{lcl}
\hline & $\begin{array}{c}\text { Placebo } \\
(n=13)\end{array}$ & \multicolumn{1}{c}{$\begin{array}{c}\text { PDX } \\
(n=16)\end{array}$} \\
\hline Before $(/ \mathrm{wk})$ & $3.5[2.5-7.0]$ & $3.0[2.5-7.0]$ \\
Ingestion week 1 (/wk) & $4.0[3.5-7.5]$ & $7.0[4.0-9.0]^{\mathrm{a}}$ \\
Ingestion week 2 (/wk) & $3.0[3.0-6.5]$ & $5.0[4.0-9.8]^{\mathrm{a}, \mathrm{b}}$ \\
Ingestion week 3 (/wk) & $4.0[3.0-7.0]$ & $8.0[3.3-10.5]^{\mathrm{a}}$ \\
Ingestion week 4 (/wk) & $5.0[3.0-7.0]$ & $8.5[6.0-12.8]^{\mathrm{a}, \mathrm{b}}$ \\
After $(/ \mathrm{wk})$ & $4.5[3.3-6.8]$ & $7.3[5.5-10.0]^{\mathrm{a}, \mathrm{b}}$
\end{tabular}

The participants who consumed polydextrose had marked changes in bowel function. Because the defecation frequency showed nonnormal distribution continuous variables were expressed as median and interquartile range (IQR).

Value: median [25th percentile-75th percentile].

${ }^{a}$ Significantly different from baseline within the same group, $p<0.05$.

${ }^{\mathrm{b}}$ Significantly different from the placebo group, $p<0.05$.

pended on the assumption that $\alpha$ error probability was $5 \%, \beta$ error probability was $20 \%$, and efficacy rates of the study and control groups were $70 \%$ and $20 \%$, respectively. Consequently, the sample size of each group was determined to be 20 cases or more.
Table 4. Stool score.

\begin{tabular}{lcc}
\hline & \multicolumn{2}{c}{ Total } \\
\cline { 2 - 3 } & $\begin{array}{c}\text { Placebo } \\
(n=13)\end{array}$ & $\begin{array}{c}\text { PDX } \\
(n=16)\end{array}$ \\
\hline Before & $3.1 \pm 0.3$ & $3.2 \pm 0.3$ \\
Ingestion & $3.2 \pm 0.2$ & $3.3 \pm 0.2$ \\
After & $3.3 \pm 0.2$ & $3.4 \pm 0.2$ \\
\hline
\end{tabular}

Value: mean \pm SD.

Score $1=$ like nuts; score $2=$ sausage-shaped; score $3=$ like a sausage or snake, smooth and soft; score $4=$ soft blobs with clear-cut edges; score $5=$ a mushy stool; score $6=$ entirely liquid.

Assuming a 20\% drop-out rate among the cases, the sample size was estimated at 12 participants per group to provide a power of $80 \%$ to detect a difference in stool frequency as the primary outcome of interest between the 2 groups, which is in agreement with a 4 -wk, randomized, triple-blind, placebo-controlled, parallel study, with a 2 -sided $\alpha$-level of 0.05 .

Statistical analysis. All results were analyzed according to the per-protocol principle. The interquartile range 


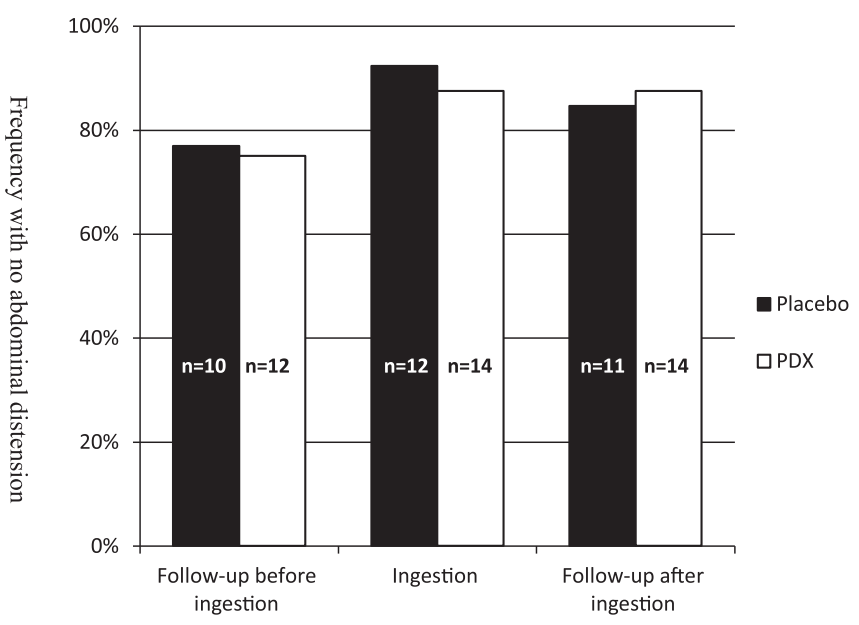

Fig. 3. Abdominal distension. There were no significant differences between groups in ratings of abdominal distension before, during, or after the trial period.

(IQR) was used to detect the presence of potential outliers. A Kolmogorov-Smirnov test was used to assess the normality of each variable. The Student's $t$-test or chi-square test was used to compare the baseline variables between groups. All values were expressed as the mean \pm standard deviation, median 25 th or 75 th percentile, or percentage, as appropriate, and $p<0.05$ was considered statistically significant. Comparisons among quartiles were assessed with one-way analysis of variance, Kruskal-Wallis, or $\chi^{2}$ test, as appropriate.

Self-reported stool consistency was recorded using a 6-point scale: $1=$ like nuts; 2 =sausage-shaped; $3=$ like a sausage or snake, smooth and soft; $4=$ soft blobs with clear cut edges; $5=$ a mushy stool; and $6=$ entirely liquid. For continuous variables, 2-group comparisons were performed with both the parametric 2-sample $t$-test and nonparametric Mann-Whitney test. Analysis of variance was used to compare groups (before comparison with after PDX intake) and the paired sample $t$-test was used to determine differences between the PDX and placebo groups.

Repeated-measures analysis of variance (ANOVA) was used to compare clinical chemistry indexes between groups. Statistical analyses were performed using SPSS 21.0 (IBM Corporation, Armonk, NY). A 2-tailed value of $p<0.05$ was considered statistically significant.

\section{Results}

A total of 50 participants was enrolled and randomized into either the PDX group $(n=25)$ or the control group $(n=25)$. Of the participants enrolled, 21 were not included for the efficacy analysis, primarily owing to protocol inclusion/exclusion violations in the placebo group $(n=12)$, and the use of treatment regimens such as laxative or phosphate binders that were violations of the protocol in the PDX group $(n=9)$. The remaining 29 patient constituted the per protocol population, the primary efficacy population for the analysis (Fig. 2 ). The mean compliance ratio for all participants was $96.4 \%$, which was sufficient. There were no adverse events or significant subjective symptoms (i.e., anorexia, abdominal discomfort, constipation, and stomach flatulence) reported by any of the participants in this study. At enrollment and analysis, the distributions of age, sex, body mass index and dialysis duration between the groups, and the stool scores and defecation frequency did not differ (Table 1).

The participants who consumed PDX had marked changes in stool frequency (Table 3). The PDX group showed significant improvements in bowel function (increased defecation frequency), while no significant change was observed in the stool score (Table 4). The PDX group had significantly increased stool frequency compared to before the PDX intake period: 7.0, 5.0, 8.0, 8.5 bowel movements per week during the PDX study period (week 1-week 4) and 7.3 bowel movements after the end of the study period ( $p<0.01$ ). In addition, this group had significantly more bowel movements during and following the study period than the control group $(p<0.05)$. There were no significant differences between groups in ratings of abdominal distension and no reports of abdominal cramps, diarrhea, or other discomforts (Fig. 3).

At the beginning and end of the study period, participants were interviewed, using a standardized questionnaire (a food frequency questionnaire based on food groups, and a modified version of the Bristol stool form scale) to assess the dietary condition, occurrence, and severity of symptoms and the acceptability of the preparations. All participants were adults with a low habitual intake of dietary fiber from baseline to the completion of the study (Table 5). The intake levels of total energy and selected nutrients were not different between the groups at baseline, with the exception of carbohydrate and potassium. While the total energy and macronutrient intake levels were unchanged during the study, paired $t$-test analysis showed that retinol activity equivalents intake was significantly increased in the PDX group $(p<0.05)$.

Clinical biochemistry indexes (e.g., measures of liver and renal function, blood electrolytes, fasting blood glucose, triglyceride, cholesterol, and serum glycoalbumin) 
Table 5. Nutrition intake.

\begin{tabular}{|c|c|c|c|c|}
\hline & & $n$ & Before & After \\
\hline \multirow[t]{2}{*}{ Energy (kcal) } & placebo & 13 & $1,440 \pm 369$ & $1,465 \pm 366$ \\
\hline & PDX & 16 & $1,624 \pm 371$ & $1,631 \pm 237$ \\
\hline \multirow[t]{2}{*}{ Protein (g) } & placebo & 13 & $50.6 \pm 17.3$ & $53.1 \pm 17.2$ \\
\hline & PDX & 16 & $55.7 \pm 8.2$ & $56.7 \pm 10.6$ \\
\hline \multirow[t]{2}{*}{ Lipid (g) } & placebo & 13 & $46.3 \pm 18.9$ & $47.1 \pm 18.5$ \\
\hline & PDX & 16 & $49.4 \pm 11.2$ & $50.5 \pm 13.6$ \\
\hline \multirow[t]{2}{*}{ Carbohydrate (g) } & placebo & 13 & $199.3 \pm 46.0$ & $201.3 \pm 43.9$ \\
\hline & PDX & 16 & $231 \pm 29.9^{a}$ & $228.7 \pm 31.9$ \\
\hline \multirow[t]{2}{*}{ Potassium (mg) } & placebo & 13 & $1,614 \pm 570$ & $1,683 \pm 573$ \\
\hline & PDX & 16 & $2,014 \pm 371^{\mathrm{a}}$ & $2,057 \pm 447^{\mathrm{a}}$ \\
\hline \multirow[t]{2}{*}{ Calcium (mg) } & placebo & 13 & $311 \pm 144$ & $359 \pm 166$ \\
\hline & PDX & 16 & $108 \pm 86$ & $418 \pm 109$ \\
\hline \multirow[t]{2}{*}{ Iron (mg) } & placebo & 13 & $5.0 \pm 1.4$ & $5.3 \pm 15$ \\
\hline & PDX & 16 & $5.8 \pm 1.1$ & $6.0 \pm 1.2$ \\
\hline \multirow[t]{2}{*}{ Salt equivalents (g) } & placebo & 13 & $6.0 \pm 2.8$ & $6.2 \pm 2.5$ \\
\hline & PDX & 16 & $8.0 \pm 3.0$ & $7.9 \pm 2.6$ \\
\hline \multirow[t]{2}{*}{ Retinol activity equivalents ( $\mu \mathrm{g})$} & placebo & 13 & $435 \pm 194$ & $448 \pm 175$ \\
\hline & PDX & 16 & $498 \pm 158$ & $547 \pm 174^{b}$ \\
\hline \multirow[t]{2}{*}{ Vitamin D $(\mu \mathrm{g})$} & placebo & 13 & $4.3 \pm 3.0$ & $4.5 \pm 2.8$ \\
\hline & PDX & 16 & $7.7 \pm 3.1$ & $6.9 \pm 2.9$ \\
\hline \multirow[t]{2}{*}{ Thiamin (mg) } & placebo & 13 & $0.72 \pm 0.30$ & $0.74 \pm 0.29$ \\
\hline & PDX & 16 & $0.71 \pm 0.12$ & $0.76 \pm 0.17$ \\
\hline \multirow[t]{2}{*}{ Riboflavin (mg) } & placebo & 13 & $0.74 \pm 0.25$ & $0.80 \pm 0.28$ \\
\hline & PDX & 16 & $0.85 \pm 0.14$ & $0.88 \pm 0.18$ \\
\hline \multirow[t]{2}{*}{ Ascorbic acid (mg) } & placebo & 13 & $62 \pm 33$ & $70 \pm 32$ \\
\hline & PDX & 16 & $87 \pm 26$ & $90 \pm 30$ \\
\hline \multirow[t]{2}{*}{ Dietary fibers (g) } & placebo & 13 & $9.6 \pm 3.3$ & $10.2 \pm 2.8$ \\
\hline & PDX & 16 & $11.3 \pm 2.6$ & $12.0 \pm 2.6$ \\
\hline
\end{tabular}

Value: mean \pm SD.

${ }^{\text {a }}$ Significantly different from the placebo group, $p<0.05$.

${ }^{\mathrm{b}}$ Significantly different from baseline within the same group, $p<0.05$.

The intake levels of total energy and selected nutrients were not different between the groups at baseline, with the exception of carbohydrate and potassium. In the present study, using paired $t$-test analysis, while the total energy and macronutrient intake levels were unchanged during the study, retinol activity equivalents intake was significantly increased in the PDX group $(p<0.05)$.

did not change significantly after PDX intake for $28 \mathrm{~d}$ (Table 6).

\section{Discussion}

Our study was a unique approach on how to improve constipation in HD patients without causing adverse events. This study in Japanese HD patients showed that supplementation with PDX increased stool frequency significantly but did not affect stool scores, and PDX had no influence on measured blood chemistry indexes. PDX intake led to some physiologic effects associated with dietary fiber. Patients on maintenance HD often show substantial reductions in quality of life (QOL). Although a laxative was taken, the combination with PDX did not result in stool formation that was too soft, and patients did not experience a feeling of fullness; therefore, this study demonstrated the potential to improve the QOL of HD patients.

To prevent constipation, a diet that is high in fiber is generally recommended. The average dietary fiber intake in the Japanese population is well below the recommended amount of $17-19 \mathrm{~g} / \mathrm{d}$ for adult males and females. Dietary fiber consumption in the USA is $12-18 \mathrm{~g} / \mathrm{d}$, which is well below the recommended adequate intake of $25 \mathrm{~g} / \mathrm{d}$ for females and $38 \mathrm{~g} / \mathrm{d}$ for males (21). In a United States study, a statistically significant difference was observed in the dietary fiber content between maintenance $\mathrm{HD}$ patients and non-dialysis control subjects $(12 \pm 6 \mathrm{~g} / \mathrm{d}$ vs. $18 \pm 11 \mathrm{~g} / \mathrm{d}, p=0.02)$ (22). Moreover, intake of total dietary fiber per day has been reported to be $5.9 \pm 2.7 \mathrm{~g}$ in Japanese HD patients (23). These data indicate that maintenance HD patients had significantly lower intakes of dietary fiber when compared with individuals not receiving dialysis.

Therefore, finding ways to increase fiber consumption in the Japanese is important to aid colonic health. Adding non-digestible, fermentable carbohydrate sources that act as dietary fiber and that can be added to new or existing food products is one way to potentially increase fiber consumption. In general, HD patients need to limit 
Table 6. Clinical data for the HD patients at baseline and after the 4-wk intervention.

\begin{tabular}{|c|c|c|c|c|c|c|}
\hline & \multicolumn{3}{|c|}{ Placebo } & \multicolumn{3}{|c|}{ PDX } \\
\hline & Before & $28 \mathrm{~d}$ & $p$ value & Before & $28 \mathrm{~d}$ & $p$ value \\
\hline BUN (mg/dL) & $52.9 \pm 19.4$ & $53.7 \pm 16.9$ & n.s. & $58.7 \pm 12.4$ & $55.0 \pm 12.9$ & n.s. \\
\hline Creatinine $(\mathrm{mg} / \mathrm{dL})$ & $9.6 \pm 3.2$ & $9.4 \pm 2.9$ & n.s. & $9.9 \pm 2.5$ & $9.8 \pm 2.2$ & n.s. \\
\hline $\mathrm{UA}(\mathrm{mg} / \mathrm{dL})$ & $6.7 \pm 0.8$ & $6.6 \pm 0.9$ & n.s. & $7.7 \pm 0.8^{\mathrm{a}}$ & $7.5 \pm 0.8^{\mathrm{a}}$ & n.s. \\
\hline $\mathrm{Na}(\mathrm{mEq} / \mathrm{L})$ & $138.9 \pm 3.8$ & $138.6 \pm 2.7$ & n.s. & $138.8 \pm 2.6$ & $137.9 \pm 3.2$ & n.s. \\
\hline $\mathrm{Cl}(\mathrm{mEq} / \mathrm{L})$ & $102.1 \pm 4.4$ & $102.0 \pm 3.2$ & n.s. & $102.3 \pm 4.3$ & $101.8 \pm 3.7$ & n.s. \\
\hline $\mathrm{K}(\mathrm{mEq} / \mathrm{L})$ & $4.7 \pm 0.8$ & $4.7 \pm 0.6$ & n.s. & $4.8 \pm 0.8$ & $5.1 \pm 0.9$ & n.s. \\
\hline Calcium (mg/dL) & $8.6 \pm 0.8$ & $8.4 \pm 0.6$ & n.s. & $8.3 \pm 0.6$ & $8.2 \pm 0.7$ & n.s. \\
\hline Phosphate (mg/dL) & $4.5 \pm 1.0$ & $4.9 \pm 1.1$ & n.s. & $5.1 \pm 0.9$ & $4.9 \pm 0.7$ & n.s. \\
\hline $\operatorname{Mg}(\mathrm{mg} / \mathrm{dL})$ & $2.6 \pm 0.5$ & $2.5 \pm 0.4$ & n.s. & $2.7 \pm 0.4$ & $2.8 \pm 0.4$ & n.s. \\
\hline $\mathrm{Fe}(\mathrm{mg} / \mathrm{dL})$ & $44.0 \pm 22.6$ & $43.9 \pm 13.7$ & n.s. & $72.0 \pm 43.9^{\mathrm{a}}$ & $63.7 \pm 48.7$ & n.s. \\
\hline Ferritin (mg/L) & $60.9 \pm 65.8$ & $47.6 \pm 33.4$ & n.s. & $62.2 \pm 63.2$ & $71.4 \pm 70.3$ & n.s. \\
\hline T-cho (mg/dL) & $161.1 \pm 24.2$ & $156.1 \pm 25.7$ & n.s. & $156.3 \pm 44.0$ & $153.1 \pm 41.6$ & n.s. \\
\hline HDL cholesterol (mg/dL) & $37.9 \pm 10.5$ & $38.3 \pm 9.8$ & n.s. & $47.1 \pm 16.5$ & $44.7 \pm 13.9$ & n.s. \\
\hline Triglyceride (mg/dL) & $98.8 \pm 27.3$ & $88.0 \pm 41.6$ & n.s. & $99.2 \pm 43.3$ & $95.9 \pm 44.9$ & n.s. \\
\hline LDL cholesterol (mg/dL) & $95.8 \pm 20.8$ & $92.8 \pm 19.7$ & n.s. & $81.6 \pm 28.9$ & $82.4 \pm 28.6$ & n.s. \\
\hline Total protein $(\mathrm{g} / \mathrm{dL})$ & $6.5 \pm 0.4$ & $6.6 \pm 0.4$ & n.s. & $6.4 \pm 0.4$ & $6.6 \pm 0.5$ & n.s. \\
\hline Albumin (g/dL) & $3.5 \pm 0.2$ & $3.5 \pm 0.3$ & n.s. & $3.6 \pm 0.2$ & $3.6 \pm 0.2$ & n.s. \\
\hline AST (IU/L) & $15.0 \pm 9.1$ & $17.0 \pm 11.2$ & n.s. & $13.5 \pm 6.8$ & $13.8 \pm 6.3$ & n.s. \\
\hline ALT (IU/L) & $10.9 \pm 5.9$ & $11.6 \pm 5.8$ & n.s. & $11.5 \pm 6.9$ & $11.4 \pm 4.8$ & n.s. \\
\hline$\gamma$-GTP (IU/L) & $17.2 \pm 9.1$ & $17.9 \pm 8.4$ & n.s. & $15.9 \pm 7.9$ & $16.3 \pm 7.7$ & n.s. \\
\hline $\mathrm{WBC}(/ \mu \mathrm{L})$ & $6,184 \pm 1,488$ & $6,272 \pm 1,443$ & n.s. & $5,026 \pm 1,067^{\mathrm{a}}$ & $5,362 \pm 1,560$ & n.s. \\
\hline $\mathrm{RBC}\left(\times 10^{4} / \mu \mathrm{L}\right)$ & $379.8 \pm 51.4$ & $380.9 \pm 51.4$ & n.s. & $370.6 \pm 47.8$ & $364.4 \pm 44.8$ & n.s. \\
\hline Hematocrit (\%) & $35.1 \pm 2.6$ & $35.5 \pm 3.6$ & n.s. & $34.5 \pm 3.1$ & $34.4 \pm 3.8$ & n.s. \\
\hline Hemoglobin (g/dL) & $10.8 \pm 0.7$ & $10.9 \pm 0.8$ & n.s. & $10.8 \pm 0.9$ & $10.7 \pm 1.1$ & n.s. \\
\hline Glucose (mg/dL) & $131.3 \pm 44.0$ & $123.1 \pm 24.8$ & n.s. & $129.1 \pm 24.3$ & $135.4 \pm 43.2$ & n.s. \\
\hline GA $(\%)$ & $14.5 \pm 10.7$ & $14.4 \pm 10.6$ & n.s. & $13.2 \pm 12.6$ & $11.5 \pm 12.4$ & n.s. \\
\hline
\end{tabular}

Two-sample $t$-test. n.s.: not significant.

Value: mean \pm SD.

${ }^{a}$ Significantly different from the placebo group.

No significant changes were observed between the baseline and the 28th day.

With the exception of UA, Fe and WBC, no significant changes were observed between the PDX group and the placebo group. Clinical biochemistry indexes (e.g., measures of liver and renal function, blood electrolytes, fasting blood glucose, triglyceride, cholesterol, and serum glycoalbumin) did not change significantly after PDX intake for 28d.

foods that are high in potassium and phosphate, which particularly indicates that they avoid eating high-fiber foods (24). The fiber-like character of PDX described in the literature suggested that the consumption of PDX would result in an increased stool weight (25). Higher gut contents stimulate intestinal peristalsis, which can reduce the fecal transit time (26). A study of Chinese subjects showed a dose-dependent increase in the stool weight after ingestion of $4 \mathrm{~g}, 8 \mathrm{~g}$, and $12 \mathrm{~g} \mathrm{PDX/d} \mathrm{(27).}$

There is debate as to whether these functional soluble fibers deliver known health benefits associated with 'traditional' dietary fibers. Additional fiber consumption can lead to increased gas production and overall gastrointestinal discomfort. Therefore, finding functional fibers that can be added to food products that minimally disturb gastrointestinal tolerance and acceptance of the product may result in increased fiber consumption by humans.

Dietary fiber ingestion, even in relatively small amounts, can lead to gastrointestinal discomfort but varies widely among individuals. Fiber is known to have positive effects on gastrointestinal health and has been determined to have a link to decreased disease risk (CHD, colorectal cancer, and breast cancer). Little research currently exists regarding functional soluble fibers such as PDX.

Overall, compliance was excellent throughout the study. There were no participants with more than $5 \%$ interdialytic weight gain/dry weight. We found that participants had observed the restriction of fluid intake. Diet records were analyzed and it was determined that the participants consumed a moderate-fiber diet (average fiber intake, $10.8 \mathrm{~g} / \mathrm{d}$ ). The amount of supplementary PDX was considered appropriate since it caused no difference in the blood analyses or nutritional intake between the 2 groups, and caused no adverse events.

The number of bowel movements in the PDX group was on the increase after the duration of PDX intake compared to before intake, and a similar trend was observed when compared to the placebo group. This 
could be because the intestinal environment was improved by PDX, which induces an increase in fecal bulk, softens stools, lowers fecal $\mathrm{pH}$, and is partially fermented in the large bowel (8-11), and which was also shown to be selectively fermented by the fecal microbiota and to have stimulated bifidobacterial growth in an in vitro study (28).

\section{Limitation}

Study limitations include the usage of laxatives, inclusion of participants with diabetes, and differences in physical activity and phosphorus binder prescription. Gastrointestinal symptoms such as constipation and diarrhea occur frequently in patients with diabetes mellitus. We were unable to ascertain the impact of increasing levels of physical activity on constipation and only evaluated the impact of self-reported "vigorous" exercise. Other limitations include the lack of data on the duration of self-reported constipation symptoms or the severity of constipation symptoms for comparison with stool frequency and stool consistency. A recall bias may also exist with the terminology used to ascertain "usual" stool consistency in this study design. Additionally, we were not able to let participants limit the usage of laxatives for constipation during the study. The possibility exists that a participant on laxatives may have been misclassified by our definition using stool consistency. Furthermore, we assume that these results included the Hawthorne effect in regards to the positive change in the main outcome for the control group.

\section{Conclusion}

The regular addition of PDX products, providing $10 \mathrm{~g}$ of total dietary fiber per day, to a HD patient diet is an efficacious alternative to increase fiber intake to recommended levels during $4 \mathrm{wk}$ of treatment. Moreover, PDX reduced the time to have a bowel movement and increased the number of weekly total bowel movements, without inducing adverse gastrointestinal symptoms such as abdominal pain or bloating, or producing diarrhea.

Consumption of PDX significantly improved stool frequency, softened the feces, and improved the ease of defecation, with no adverse effects. Therefore, daily intake of $10 \mathrm{~g}$ PDX improved physiologic function without adverse effects.

\section{Competing interests}

The authors report no conflicts of interest in this work.

\section{Authors' contributions}

The authors' responsibilities were as follows: M.S., conception, design of the study, data interpretation, statistical analysis and writing of the manuscript; N.N., contribution to the experimental design, data interpretation and blinding; M.S., S.G. and N.N., trial coordination; S.G., dietary fiber investigation; K.I., T.T., T.A., H.K. and T.O., contribution to the experimental design and data interpretation. All authors reviewed and agreed on the final version of the manuscript.

\section{Acknowledgments}

We thank the patients who participated in this study, and colleagues who provided statistical assistance. We thank Sayama Satoko, who performed the library searches. We also thank the Orihiro Co., Ltd.

\section{REFERENCES}

1) Trowell HC, Burkitt DP. 1975. Concluding considerations. In: Refined Carbohydrate Foods and Disease (Burkitt DP, Trowell HC, eds), p 333-345. Academic Press, London.

2) Vuksan V, Jenkins AL, Jenkins DJA, Rogovik AL, Sievenpiper JL, Jovanovski E. 2008. Using cereal to increase dietary fiber intake to the recommended level and the effect of fiber on bowel function in healthy persons consuming North American diets. Am J Clin Nutr 88: 1256-1262.

3) Frost GS, Brynes AE, Dhillo WS, Bloom SR, McBurney MI. 2003. The effects of fiber enrichment of pasta and fat content on gastric emptying, GLP-1, glucose, and insulin responses to a meal. Eur J Clin Nutr 57: 293-298.

4) Caswell S, Anderson AS, Steele RJC. 2009. Bowel health to better health: a minimal contact lifestyle intervention for people at increased risk colorectal cancer. $\mathrm{Br} J \mathrm{Nutr}$ 102: 1541-1546.

5) Hongisto SM, Paajanen L, Saxelin M, Korpela R. 2006. A combination of fibre-rich rye bread and yoghurt containing Lactobacillus GG improves bowel function in women with self-reported constipation. Eur J Clin Nutr 60: 319-324.

6) Mann JI, Cummings JH. 2009. Possible implications for health of the different definitions of dietary fibre. Nutr Metab Cardiovasc Dis 19: 226-229.

7) Pfizer Inc. 1978. Polydextrose food additive petition. Pfizer Inc, New York (FDA petition 9A3441).

8) Nakagawa Y, Okamatsu H, Fujii Y. 1990. Effects of Polydextrose feeding on the frequency and feeling of defecation in healthy adult female volunteers. J Jpn Soc Nutr Food Sci 43: 95-101.

9) Endo K, Kumemura M, Nakamura K, Fujisawa T, Suzuki K, Benno Y, Mitsuoka T. 1991. Effects of high cholesterol diet and polydextrose supplementation on the microflora, bacterial enzyme activity, putrefactive products, volatile fatty acid (VFA) profile, weight, and $\mathrm{pH}$ of the feces in healthy volunteers. Bifidobacteria Microflora 10: 53-64.

10) Achour L, Flourie B, Briet F, Pellier P, Marteau P, Rambaud JC. 1994. Gastrointestinal effects and energy value of polydextrose in healthy nonobese men. Am J Clin Nutr 59: 1362-1368.

11) Wang X, Gibson GR. 1993. Effects of the in vitro fermentation of oligofructose and inulin by bacteria growing in the human large intestine. J Appl Bacteriol 75: $373-380$.

12) Lindberg G, Hamid SS, Malfertheiner P, Thomsen OO, Fernandez LB, Garisch J, Thomson A, Goh KL, Tandon R, Fedail S, Wong BC, Khan AG, Krabshuis JH, LeMair A. 2011. World gastroenterology organisation global guideline: Constipation-A global perspective. J Clin Gastroenterol 45: 483-487.

13) Bove A, Pucciani F, Bellini M, Battaglia E, Bocchini R, Altomare DF, Dodi G, Sciaudone G, Falletto E, Piloni 
V, Gambaccini D, Bove V. 2012. Consensus statement AIGO/SICCR: diagnosis and treatment of chronic constipation and obstructed defecation (part I: diagnosis). World J Gastroenterol 18: 1555-1564.

14) Lee EJ, Warden S. 2011. A qualitative study of quality of life and the experience of complementary and alternative medicine in Korean women with constipation. Gastroenterol Nurs 34: 118-127.

15) Wu MJ, Chang CS, Cheng $\mathrm{CH}$, Chen $\mathrm{CH}$, Lee WC, Hsu YH, Shu KH, Tang MJ. 2004. Colonic transit time in long-term dialysis patients. Am J Kidney Dis 44: 322 327.

16) Murtagh FE, Addington-Hall J, Higginson IJ. 2007. The prevalence of symptoms in end-stage renal disease: a systematic review. Adv Chronic Kidney Dis 14: 82-99.

17) Hammer J, Oesterreicher C, Hammer K, Koch U, Traindl O, Kovarik J. 1998. Chronic gastrointestinal symptoms in hemodialysis patients. Wien Klin Wochenschr 110: 287-291.

18) Lewis SJ, Heaton KW. 1997. Stool form scale as a useful guide to intestinal transit time. Scand J Gastroenterol 32: 920-924.

19) Heaton KW, O'Donnell LJ. 1994. An office guide to whole-gut transit time. Patients' recollection of their stool form. J Clin Gastroenterol 19: 28-30.

20) Yoshimura Y, Takahashi K. 2010. "Excel-eiyou-kun" add-in software "FFQg." Ver. 3.0, Kenpakusha, Tokyo.

21) Trumbo T, Schlicker S, Yates AA, Poos M. 2002. Dietary reference intakes for energy, carbohydrates, fiber, fat, fatty acids, cholesterol, protein, and amino acids. J Am
Diet Assoc 102: 1621-1630.

22) Kamyar K, Joel DK, Sunaina D, Donald B, Gladys B. 2002. Food intake characteristics of hemodialysis patients as obtained by food frequency questionnaire. $J$ Ren Nutr 12: 17-31.

23) Yasuda G, Shibata Y, Takizawa T, Ikeda Y, Tokita Y, Umemura S, Tochikubo O. 2002. Prevalence of constipation in continuous ambulatory peritoneal dialysis patients and comparison with hemodialysis patients. Am J Kid Dis 39: 1292-1299.

24) Sutton D, Dumbleton S, Allaway C. 2007. Can increased dietary fibre reduce laxative requirement in peritoneal dialysis patients? J Ren Care 33: 174-178.

25) Probert HM, Apajalhti JH, Rautonen N, Stowell J, Gibson GR. 2004. Polydextrose, lactitol, and fructo-oligosaccharide fermentation by colonic bacteria in a threestage continuous culture system. Appl Environ Microbiol 70: $4505-4511$.

26) Cummings JH, Macfarlane GT, Englyst HN. 2001. Prebiotic digestion and fermentation. Am J Clin Nutr 73: 415-420.

27) Zhong J, Luo B, Xiang M, Liu H, Zhai Z, Wang T, Stuart ASC. 2000. Studies on the effects of polydextrose intake on physiologic functions in Chinese people. Am J Clin Nutr 72: 1503-1509.

28) Makivuokko H, Kettunen H, Saarinen M, Kamiwaki T, Yokoyama Y, Stowell L, Rautonen N. 2007. The effect of cocoa and polydextrose on bacterial fermentation in gastrointestinal tract simulations. Biosci Biotechnol Biochem 71: 1834-1843. 